\title{
Médiévales
}

Langues, Textes, Histoire

49 | automne 2005

La paroisse, genèse d'une forme territoriale

\section{Les recherches récentes sur la formation des paroisses en Angleterre : similitudes et différences} avec la France

Traduit de l'anglais par Bruno Laurioux

Recent work on parish formation in England: some comparisons and contrasts

with France

John Blair

\section{(2) OpenEdition}

Édition électronique

URL : https://journals.openedition.org/medievales/1267

DOI : 10.4000/medievales. 1267

ISSN : $1777-5892$

Éditeur

Presses universitaires de Vincennes

Édition imprimée

Date de publication : 1 décembre 2005

Pagination : 33-44

ISBN : 2-84292-177-1

ISSN : 0751-2708

Référence électronique

John Blair, «Les recherches récentes sur la formation des paroisses en Angleterre : similitudes et différences avec la France », Médiévales [En ligne], 49 | automne 2005, mis en ligne le 05 mars 2008 , consulté le 24 avril 2022. URL : http://journals.openedition.org/medievales/1267 ; DOI : https:// doi.org/10.4000/medievales. 1267

Ce document a été généré automatiquement le 24 avril 2022.

Tous droits réservés 


\section{Les recherches récentes sur la formation des paroisses en Angleterre : similitudes et différences avec la France}

Traduit de l'anglais par Bruno Laurioux

Recent work on parish formation in England : some comparisons and contrasts with France

John Blair

\section{NOTE DE L'ÉDITEUR}

Les figures mentionnées dans cet article sont consultables dans la version imprimée du numéro 49.

En 2004, lorsque se tint la journée d'études de Tours qui a donné naissance à ce numéro de Médiévales, mon propre livre sur les églises locales dans l'Angleterre du haut Moyen Âge - résultat lui-même de longues années de recherches - était sous presse ${ }^{1}$. C'était donc pour moi le pire moment possible pour dire quelque chose de neuf. J'espère néanmoins que quelques réflexions (suscitées par ce que j'ai entendu à Tours) sur la manière dont les récentes approches de chaque côté de la Manche convergent ou divergent peuvent représenter un utile complément aux autres contributions de ce dossier. Jusqu'à tout récemment, la plupart des archéologues anglais, et même beaucoup d'historiens anglais n'ont tenu presque aucun compte des avancées conceptuelles et du grand développement des fouilles systématiques en France. Pour leur part - et bien que ce volume manifeste la rapidité avec laquelle les conceptions sont en train de changer - les chercheurs français ont souvent appliqué aux régions culturellement différentes de la France et à la complexité des réalités du terrain un 
modèle théorique assez statique de la formation des paroisses, dérivé avant tout de Grégoire de Tours et des prescriptions de conciles ecclésiastiques.

Désormais nous commençons enfin à nous parler. Les chercheurs français ont pris davantage conscience que les structures appropriées (en théorie et en pratique) aux territoires situés au sud des bassins de la Seine et de la Loire ne s'appliquaient pas nécessairement aux régions du Nord de la France; de même, les perspectives offertes par l'étude de ces régions septentrionales sont maintenant prises plus au sérieux en Angleterre. Il est aussi frappant de voir comment les modèles d'évolution (par exemple sur les origines de l'inhumation dans le cimetière de l'église et de la formation des limites paroissiales) non seulement changent des deux côtés de la Manche mais changent dans la même direction. Dans le même temps, les différences, qui continuent à apparaitre comme authentiques, ressortent avec plus de clarté. Il serait inutile d'essayer ici de résumer mon livre. J'examinerai plutôt - et brièvement - quelques domaines dans lesquels la recherche anglaise converge avec les thèmes et les problèmes abordés dans ce numéro de Médiévales.

Une organisation épiscopale romaine?

3 La première mission officielle en Angleterre, celle de saint Augustin dans le royaume du Kent en 597, fut envoyée par Rome. Les autres missionnaires qui suivirent durant un demi-siècle, à l'exception des Irlandais, venaient soit d'Italie soit de Gaule. Ils auraient tout naturellement cherché à introduire les structures pastorales centralisées et dirigées par des évêques qu'ils connaissaient dans leurs pays d'origine. De fait, le pape Grégoire le Grand avait projeté un dense réseau diocésain: vingt-quatre sièges ordinaires dirigés par deux métropolitains ${ }^{2}$. Mais l'ancienne province de Britannia était devenue entre-temps très différente du Sud de la Gaule ou de l'Italie du Nord: la population avait chuté, l'économie rurale et la vie urbaine s'étaient totalement effondrées, et les preuves d'une continuité des structures civiques ou ecclésiastiques dans les royaumes anglo-saxons sont minces ou inexistantes. Comment concilier alors la théorie d'une Église sous contrôle des évêques avec cette réalité politique et économique?

4 Il existe quelques maigres indices de l'existence d'églises baptismales sous-diocésaines, analogues aux pievi, dans le minuscule diocèse de Cantorbéry. Dans les années 620, Paulin, un membre de la mission d'Augustin, s'est tourné vers le royaume beaucoup plus vaste de Northumbrie, dans le Nord, en a converti le roi, et a entrepris d'y fonder également des églises pastorales; en des termes qui rappellent Grégoire de Tours et les conciles gaulois (et qui pourraient dériver d'un document de Cantorbéry), Bède le Vénérable parle des basilicae, oratoria et baptisteria de Northumbrie ${ }^{3}$. Mais tous les témoignages suggèrent que ce système paroissial naissant de type romain disparut en quelques décennies - et probablement était-il condamné dès le départ. Là où Grégoire le Grand avait prévu vingt-six sièges, on comptait seulement trois évêques en 669 et encore seulement une douzaine en 690 . Après 650 , en fait, l'avenir repose sur les grandes et diverses communautés religieuses que l'on peut qualifier, au sens le plus large du terme, de monastiques.

5 Le triomphe du modèle monastique dans les royaumes anglo-saxons doit certainement quelque chose à l'Irlande, mais l'idée de deux systèmes distincts et opposés - l'un " romain » et l'autre «celtique »- est fausse et peu utile. Nous devons plutôt situer notre regard au niveau d'une large région de l'Europe du Nord-Ouest dans laquelle le pouvoir fondé sur la cité s'est effondré ou bien n'a jamais existé, zone qui était mieux 
adaptée aux structures plus décentralisées et familiales des monasteria (cf. fig. 1). Il y a quarante ans, Jean-François Lemarignier a proposé de diviser la France nouvellement christianisée en « Gaule conciliaire » dans le Sud et « Gaule monastique » dans le Nord 4 . Quoique peut-être trop rigide, cette distinction a le mérite d'attirer l'attention sur une frontière culturelle du haut Moyen Âge : il y a des raisons de penser que la vallée de la Loire représentait une limite plus essentielle que la Manche. Aux VII ${ }^{\mathrm{e}}$ et VIII ${ }^{\mathrm{e}}$ siècles, une série de liens économiques, dynastiques, culturels et religieux ont uni les monastères (singulièrement les établissements féminins) du Nord de la France et ceux des royaumes anglo-saxons ${ }^{5}$. Dans ce monde de puissantes parentèles et de systèmes économiques décentralisés qui s'étendait des deux côtés de la Manche, le nouveau monachisme représentait un fort lien social et un vecteur pour les idées nouvelles.

Le nord-ouest de l'Europe chrétienne dans les années 660 : représentation schématique du contraste entre les zones où la vie urbaine et la culture romaine se maintiennent (avec une intensité qui diminue du sud vers le nord), et les zones où domine une organisation ecclésiastique fondée sur le

monachisme (J. Blair, The Church in Anglo-Saxon Society, Oxford, 2005, fig. 6, reproduite avec l'aimable autorisation de Oxford University Press).

Un paysage religieux monastique?

6 Le monastère a-t-il été aussi le principal vecteur de la pastorale des laïcs ? Cette question a été très débattue et les historiens anglais sont toujours en désaccord sur la solution à lui apporter. Selon moi, le mot latin monasterium (vieil-anglais mynster) était utilisé de manière très large, pour couvrir un grand nombre et une large gamme d'établissements dont le personnel ne se limitait pas nécessairement au groupe restreint (et habituellement de niveau aristocratique) des moniales et des moines. Sur les marges de cet ensemble on peut vaguement discerner des groupes de prêtres et de clercs de statut inférieur qui pourraient avoir été les principaux interlocuteurs de la population paysanne: quelques textes brefs mais capitaux montrent qu'ils s'acquittaient envers eux de tâches de nature pastorale $^{6}$. De tels minsters (terme qui a l'avantage de moderniser mynster tout en évitant les connotations plus étroites du mot « monastère ») furent fondés en très grand nombre durant la période 670-740, de sorte que, au milieu du viII siècle, la plupart des habitants des lowlands anglais se trouvaient probablement à une distance raisonnablement proche de l'un d'entre eux ${ }^{7}$. Dès le $\mathrm{x}^{\mathrm{e}}$ siècle au plus tard, beaucoup d'entre eux étaient devenus les centres de grands territoires proto-paroissiaux et recevaient une série de paiements réguliers en nature ou en espèces de la part des habitants de ces territoires. Des indices laissent penser que, au moins dans certains cas, les origines de ces territoires remontent à avant $800^{8}$. Quelque centralisée qu'ait été l'activité - puisque les prêtres étaient basés dans des sites ecclésiastiques complexes au centre de territoires de taille importante-, le monastère semble avoir été contrôlé davantage par les minsters que par les évêques (sauf lorsque ces minsters étaient eux-mêmes propriétés épiscopales).

7 Ce système paroissial à direction monastique, si dissemblable de celui qui régnait en France au sud de la Loire, peut ne pas avoir été très différent de la situation de régions plus septentrionales, telle la Bretagne avec ses églises de plebs attestées par les chartes de Redon ${ }^{9}$. Il est toutefois probable que les «paroisses monastiques» devaient leur cohérence aux systèmes remarquablement réguliers de division, d'estimation et d'imposition des terres séculières sur lesquels les anciens royaumes anglo-saxons étaient fondés. Les termes latins que Bède le Vénérable emploie pour les redevances ecclésiastiques - tributum, pondus, vectigal - appartiennent au vocabulaire de la fiscalité royale, et le principal mot en vieil-anglais pour une paroisse-mère - hyrness - signifie 
simplement « seigneurie ». Il y a une autre raison pour laquelle l'encadrement pastoral assuré par les minsters pourrait avoir été exceptionnellement efficace: jusqu'au $\mathrm{x}^{\mathrm{e}}$ siècle, il semble y avoir eu très peu d'églises locales fondées par des seigneurs et susceptibles de concurrencer ce système. Tandis que la France comptait de nombreuses églises domaniales, repérables par exemple grâce à l'archéologie des «sépultures privilégiées " mérovingiennes ou dans les documents carolingiens, les témoignages écrits, en Angleterre, se limitent à quelques récits de miracles de Bède le Vénérable et les indices archéologiques sont pratiquement absents ${ }^{10}$.

8 Le premier système paroissial anglo-saxon pourrait donc avoir été exceptionnellement efficace et il semble s'être renforcé même lorsque la richesse et le prestige culturel des minsters déclinèrent durant les $\mathrm{Ix}^{\mathrm{e}}$ et $\mathrm{x}^{\mathrm{e}}$ siècles. Ce fut seulement après 950 qu'il fut soumis à des tensions, face au changement économique et à une vague longuement retardée de fondations d'églises locales.

Les églises locales et la « mutation féodale »

9 L'Angleterre n'a pas expérimenté la fragmentation du pouvoir politique et de la justice associée à la « mutation féodale », mais durant les $\mathrm{x}^{\mathrm{e}}$ et $\mathrm{XI}^{\mathrm{e}}$ siècles, la mise en place d'un pouvoir économique plus décentralisé et l'imposition de l'ordre seigneurial sur les paysans y suivirent des modèles similaires à ce que l'on a observé pour la France ${ }^{11}$. En même temps que s'agglomèrent les villages, que se forment des systèmes agraires plus intensifs et que se construisent des manoirs, commencent à être fondées de petites églises rurales, qui deviendront finalement les églises paroissiales de l'Angleterre de la fin du Moyen Âge. Les témoins archéologiques convergent maintenant avec les témoignages écrits pour offrir une chronologie assez claire de ce processus. Tout comme des sites d'églises sont pour la première fois mentionnés dans des descriptions de confins depuis les années 950, et tout comme les églises situées sur des domaines seigneuriaux le sont dans une loi des années 960 , de même les premières phases (en général des constructions en bois) des églises paroissiales révélées par les fouilles peuvent être régulièrement datées d'entre le milieu du $\mathrm{x}^{\mathrm{e}}$ et le milieu du XI $\mathrm{XI}^{\mathrm{e}}$ siècle. Ce phénomène semble avoir véritablement transformé le paysage religieux de l'Angleterre qui n'avait compté jusque là que peu de bâtiments ecclésiastiques, à l'exception des minsters et de leurs dépendances, et qui s'enrichit d'un grand nombre de ces bâtiments en seulement deux ou trois générations ${ }^{12}$. Après l'an mil, de plus en plus d'églises furent dotées de leurs propres possessions foncières et rebâties en pierres.

Cette évolution a paru jusqu'ici très différente de celle que l'on a observée en France, où la période située après 950 a traditionnellement été vue comme une phase tardive dans la mise en place des églises locales. Mais la recherche actuelle, comme ce volume le montre, semble remettre en question les prétendues origines anciennes de beaucoup d'églises françaises et accorder une nouvelle importance à la croissance des $\mathrm{x}^{\mathrm{e}}$ et $\mathrm{XI}^{\mathrm{e}}$ siècles ${ }^{13}$. En Angleterre même, on observe de forts contrastes régionaux, entre les régions orientales, où les églises locales étaient clairement fréquentes dès 1000, et les régions plus occidentales où ces églises étaient peut-être encore relativement rares en 1100 - et, par certains côtés, les premières (avec leur culture influencée par les Vikings) ont plus en commun avec la Scandinavie, où les petites églises proliférèrent également à compter du début du $\mathrm{XI}^{\mathrm{e}}$ siècle. Une fois de plus, il n'est guère utile de concevoir la France ou l'Angleterre comme des entités indépendantes et homogènes : comme pour la construction des châteaux, il y eut une vague de constructions d'églises qui s'étendit 
depuis le cœur de l'Europe jusqu'à ses périphéries septentrionales dans un laps de temps relativement court.

Identité paroissiale et organisation des inhumations

11 Vers 1150 au plus tard, l'Angleterre était divisée en paroisses locales, c'est-à-dire en entités stables aux limites reconnues, par-dessous la protection d'évêques aux pouvoirs croissants et revendiquant l'exclusivité, en matière religieuse, sur les fidèles vivant dans ces limites. C'était le résultat d'évolutions qui à l'évidence avaient commencé au début $\mathrm{du} \mathrm{XI}{ }^{\mathrm{e}}$ siècle, mais qu'il est étrangement difficile de repérer dans les sources écrites. Les codes de lois anglo-saxonnes s'occupent de défendre les droits des minsters plutôt que des églises locales et le vocabulaire anglais pour les paroissiens et leurs obligations est demeuré dans une large mesure celui de la seigneurie laïque. Un indice nous est offert par le terme complexe cyricsocn (littéralement « fait de se rapprocher d'une église »), qui combine l'idée d'assister à la messe (devoir d'ordre spirituel) et celle de fréquenter l'église domaniale (un devoir lié au système manorial) ${ }^{14}$. Cyricsocn semble avoir été le mot normal pour désigner les paroisses locales qui ont émergé durant le $\mathrm{XI}^{\mathrm{e}}$ siècle et cela illustre à quel point la discipline chrétienne en vint à être formulée en termes de possession foncière et de discipline seigneuriale.

Dans la pratique, les paroisses, telles qu'elles émergent des sources après le XII siècle, équivalaient parfois aux manoirs, villages ou communautés agraires, et parfois les recouvraient de manière complexe et arbitraire ${ }^{15}$. Comme dans les cas qu'a étudiés Samuel Leturcq ${ }^{16}$, les communautés paroissiales d'Angleterre semblent avoir pris forme en réponse à des identités communautaires de nature non religieuse.

Les effets du resserrement de la discipline paroissiale sur la vie des simples paroissiens restent largement invisibles, mais dans deux domaines importants - les rites d'entrée et de sortie de la communauté chrétienne - les indices matériels peuvent nous renseigner. En Angleterre (et, si l'on en juge par les récentes recherches de Patrick Drake, plus généralement en Europe du Nord), les fonts baptismaux en pierre sont communs depuis le $\mathrm{XII}^{\mathrm{e}}$ siècle et même la fin du $\mathrm{XI}^{\mathrm{e}}$ siècle, mais ils sont rarement plus anciens ${ }^{17}$. Bien que ce phénomène puisse être en partie dû à des raisons techniques, telles que le remplacement des fonts en bois ou en plomb par des fonts en pierre, il suggère que le rituel du baptême s'est stabilisé dans les nouvelles églises locales et a été «monumentalisé » dans des structures inamovibles. Si le contrôle sur ce rituel capital d'initiation a été un pas important sur la route menant des églises domaniales aux églises locales, cet élément suggère que le nouvel ordre des choses était encore en train de se renforcer autour de 1100.

Nos connaissances sur l'évolution des pratiques d'inhumation ont progressé rapidement et promettent de le faire encore plus vite maintenant que les archéologues des deux côtés de la Manche accordent enfin toute l'attention qu'elles méritent aux tombes postérieures à l'abandon de la pratique des dépôts funéraires. Plutôt qu'un transfert immédiat depuis les nécropoles "païennes" vers les cimetières d'églises, nous pouvons désormais repérer un processus d'évolution durant lequel les chrétiens furent inhumés de manière très variée: dans la nécropole communautaire traditionnelle ou bien dans des cimetières familiaux, dans des groupes informels de tombes non associés à des églises, dans les cimetières des minsters ou bien dans ceux des nouvelles paroisses. C'est avant tout l'usage croissant de la datation au radiocarbone qui a permis d'assigner à des groupes inclassables de tombes orientées et dépourvues de mobilier - préalablement attribuées à la période romaine ou même post-médiévale - 
une date comprise entre le $\mathrm{VII}^{\mathrm{e}}$ et le $\mathrm{XI}^{\mathrm{e}}$ siècles ${ }^{18}$. Comme Hélène Noizet l'observe pour Tours ${ }^{19}$, le repérage croissant de petits cimetières dispersés et d'apparence informelle indique un phénomène plus complexe, plus diffus et plus évolutif que le modèle traditionnel et bien ordonné de l'inhumation contrôlée à tous les niveaux par l'Église.

Ainsi l'inhumation chrétienne n'était-elle pas nécessairement une inhumation dans un cimetière paroissial : la création de parcelles foncières délimitées et d'usage exclusif, dans lesquelles tous les fidèles étaient contraints de se faire enterrer, a peut-être moins à voir avec la conviction religieuse qu'avec le resserrement des systèmes de contrôle, aussi bien ecclésiastique que temporel. Grâce surtout aux études indépendantes, mais se renforçant l'une l'autre, de Cécile Treffort et d'Helen Gittos, il est devenu clair que l'établissement de cimetières d'église à l'intérieur de limites stables, leur consécration par des évêques, l'exclusion des criminels et autres indésirables de cet espace sanctifié, furent des innovations du $\mathrm{x}^{\mathrm{e}}$ siècle ${ }^{20}$. Les fouilles en ont désormais mis en évidence de bons exemples en Angleterre, notamment à Raunds (Northants), où a été retrouvé intact l'ensemble des niveaux de l'église, des limites du cimetière et des inhumations entre 950 et 1100 environ ${ }^{21}$. Tout comme les communautés séculières étaient alors plus rigoureusement définies par la loi, la seigneurie et la nécessité économique, de même, reposer dans un cimetière (souvent attaché à l'église d'un seigneur) devint à la fois le droit et le devoir des communautés de fidèles.

Entre le $\mathrm{x}^{\mathrm{e}}$ et le $\mathrm{xIII}^{\mathrm{e}}$ siècles, en France comme en Angleterre, les cimetières ecclésiastiques qui existaient déjà furent parfois reconfigurés par la création de limites entourant un espace plus étroit. Ce phénomène de contraction du cimetière nous fournit une conclusion appropriée car il illustre à la fois les similitudes dans les modèles d'évolution et l'importance d'une collaboration entre chercheurs des deux côtés de la Manche. Observant que la plupart des cimetières « rétrécis » d'Angleterre étaient adossés à des minsters, j'ai relié cette contraction au redéploiement des inhumations depuis les minsters vers les nouveaux cimetières des églises locales, redéploiement qui aurait réduit l'espace que les premiers devaient consacrer aux tombes. Dans la tradition d'une historiographie française qui met davantage l'accent sur l'évolution des relations spatiales entre les vivants et les morts, Élisabeth ZadoraRio a associé le même processus (qu'elle observait par exemple à Rigny, Indre-et-Loire) au souci de séparer le sacré du profane, également observable dans le fait d'exclure des cimetières les habitations et les activités domestiques ${ }^{22}$. Les deux explications ne sont pas nécessairement exclusives l'une de l'autre, et il demeure possible que les causes qui ont joué en Angleterre aient été vraiment différentes de celles qui jouèrent en France. Néanmoins, cet exemple illustre très clairement le besoin de considérer en parallèle les phénomènes et les modèles d'explication. Des rencontres, comme celle qui a donné naissance au présent numéro de Médiévales, doivent devenir la règle plutôt que l'exception.

17 [Le traducteur tient à chaleureusement remercier Alban Gautier et Élisabeth ZadoraRio pour leurs précieuses corrections.] 


\section{NOTES}

1. J. Blair, The Church in Anglo-Saxon Society, Oxford, 2005 (désormais abrégé CASS). On trouvera dans ce livre un exposé bien plus développé des thèmes abordés ici, ainsi que les références bibliographiques.

2. Bede, Historia Ecclesiastica Gentis Anglorum, I, 29, B. Colgrave, R. A. B. Mynors éd., Oxford, 1969, p. 104.

3. Ibid., II, 14, p. 188 ; CASS, p. 68-72. Pour le vocabulaire, comparer avec C. Delaplace, «Les origines des églises rurales ( $\mathrm{v}^{\mathrm{e}}$-vie siècles). À propos d'une formule de Grégoire de Tours ", Histoire et sociétés rurales, 18, 2002, p. 11-40.

4. J.-F. Lemarignier, «Quelques Remarques sur l'organisation ecclésiastique de la Gaule du viie à la fin du ix ${ }^{\mathrm{e}}$ siècle ", dans Settimane di Studio del centro italiano di Studi sull'Alto Medioevo, 13, Spolète, 1966, p. 451-486. Pour les aspects économiques de cette frontière, voir S. Loseby, « Gregory's cities : urban functions in sixth-century Gaul », dans I. Wood éd., Franks and Alamanni in the Merovingian Period, Woodbridge, 1998, p. 239-284.

5. S. Lebecq, "The role of monasteries in the systems of production and exchange of the Frankish world between the seventh and the beginning of the ninth centuries ", dans The Long Eighth Century, I. L. Hansen, C. Wickham éd., Leyde, 2000, p. 121-148 ; B. Yorke, Nunneries and the Anglo-Saxon Royal Houses, Londres, 2003, p. 17-36, 147-150.

6. CASS, p. 160-5 ; Bede, op. cit., III, 26, p. 310.

7. CASS, p. 80-91, 149-152.

8. CASS, p. 153-160.

9. W. Davies, «Priests and rural communities in east Brittany in the ninth century », Études celtiques, 20, 1983, p. 177-197.

10. CASS, p. 118-21 ; Bede, op. cit., V, 4-5, p. 462-4. Cf. S. Burnell, E. James, « The archaeology of conversion on the Continent in the sixth and seventh centuries : some observations and comparisons with Anglo-Saxon England ", dans R. Gameson éd., St Augustine and the Conversion of England, Stroud, 1994, p. 83-106.

11. T. Reuter et alii, "Debate : the "Feudal Revolution" ", Past and Present, 155, 1997, p. 177-225 ; D. Bates, "England and the "Feudal Revolution" ", dans Settimane di Studio del Centro Italiano di Studi sull'Alto Medioevo, 47, Spolète, 2000, p. 611-646. Voir aussi Médiévales, 21, automne 1991 (L'an mil : rythmes et acteurs d'une croissance) et 37, automne 1999 (L'an mil en 2000).

12. CASS, p. 374-407.

13. J'avais déjà commencé à prendre conscience de cette évolution (cf. CASS, p. 422 n.), mais à la lumière de la journée d'études de Tours, je soulignerai ce point plus fortement aujourd'hui.

14. CASS, p. 427-433.

15. CASS, p. 498-504.

16. Cf. l'article de Samuel Leturcq dans ce numéro de Médiévales.

17. CASS, p. 459-63 ; P. Drake, The Romanesque Fonts of Northern Europe and Scandinavia, Woodbridge, 2002.

18. C. Treffort, L'Église carolingienne et la Mort, Lyon, 1996, p. 168-170 ; D. M. Hadley, "Burial practices in northern England in the late Anglo-Saxon period ", dans S. Lucy, A. Reynolds éd., Burial in Early Medieval England and Wales, Londres, 2002, p. 209-28 ; CASS, p. 243-244, 465. 
19. Cf. l'article d'Hélène Noizet dans ce numéro de Médiévales.

20. C. Treffort, «Consécration de cimetière et contrôle épiscopal des lieux

d'inhumation au $\mathrm{X}^{\mathrm{e}}$ siècle ", dans M. Kaplan éd., Le Sacré et son inscription dans l'espace à

Byzance et en Occident, Paris, 2001, p. 285-299 ; H. Gittos, « Creating the sacred : Anglo-

Saxon rites for consecrating cemeteries ", dans Burial in Early Medieval England and

Wales, p. 195-208; CASS, p. 465.

21. A. Boddington, Raunds Furnells : the Anglo-Saxon Church and Churchyard, London, 1996 ; CASS, p. 390 n., pour d'autres cas.

22. CASS, p. 467 ; É. Zadora-Rio, « The making of churchyards and parish territories in the early medieval landscape of France and England in the 7th-12th centuries : a

reconsideration ", Medieval Archaeology, 47, 2003, p. 1-19.

\section{RÉSUMÉS}

Cet article compare la formation des paroisses en Angleterre et en France. Le modèle d'un système mis en place par l'autorité épiscopale - fondé essentiellement sur les textes des conciles et les sources concernant la Touraine - convient moins bien à l'Angleterre et au Nord de la France qu'aux régions situées au sud de la Loire: au cours des années 650-850, la culture essentiellement monastique de l'Angleterre la rapproche de la "Gaule monastique » de J.-F. Lemarignier. Les premières paroisses anglaises se sont formées autour des centres monastiques régionaux (minsters), en s'adaptant aux structures de domination foncière, d'imposition et d'identité communautaire. C'est seulement à partir de 900, et de la fragmentation des grands domaines fonciers, que ces "paroisses-mères » ont été subdivisées à leur tour et ont donné naissance à de nouvelles paroisses, d'origine seigneuriale: un processus qui est probablement plus proche qu'on ne le croyait de celui qui s'est produit dans certaines régions de la France du Nord. Cette nouvelle identité paroissiale s'est traduite par un contrôle accru des inhumations et la mise en place, autour des minsters comme des églises locales, de cimetières consacrés et délimités.

Recent work on parish formation in England: some comparisons and contrasts with France. This paper compares parish formation in England and France. The model of an episcopally-directed system based primarily on Church councils and the sources for the Touraine - is less appropriate to England and northern France than to regions south of the Loire: in its primarily monastic ecclesiastical culture during c.650-850, England resembled J.-F. Lemarignier's «Gaule monastique ». The first English parishes developed around regional monastic centres ( «minsters »), and reflected patterns of land-lordship, taxation and community identity. Only after 900, with the fragmentation of land-holding, did these «mother-parishes» fragment in turn into the local parishes of manorial churches : a process which may have been closer to parts of northern France than has been thought. One sign of this new parish identity is a more controlled burial practice, with the rise of consecrated and bounded graveyards at both minsters and local churches. 
INDEX

Mots-clés : paroisse, monastère, encadrement pastoral, sépulture

Keywords : parish, monastic, pastoral care, burial 